\title{
Observing Optical Plasmons on a Single Nanometer Scale
}

SUBJECT AREAS:

NANOPHOTONICS AND

PLASMONICS

OPTICAL PHYSICS

OPTICAL TECHNIQUES

Received

4 September 2013

Accepted

27 January 2014

Published

21 February 2014

Correspondence and requests for materials should be addressed to M.C. (moshik. cohen80@gmail.com)

\author{
Moshik Cohen ${ }^{1,2}$, Reuven Shavit ${ }^{2}$ \& Zeev Zalevsky ${ }^{1,3}$
}

${ }^{1}$ Faculty of Engineering, Bar-llan University, Ramat-Gan 52900, Israel, ${ }^{2}$ Department of Electrical and Computer Engineering, Ben-Gurion University of the Negev, Beer-Sheva 84 105, Israel, ${ }^{3}$ Bar-llan Institute for Nanotechnology \& Advanced Materials, RamatGan 52900, Israel.

The exceptional capability of plasmonic structures to confine light into deep subwavelength volumes has fashioned rapid expansion of interest from both fundamental and applicative perspectives. Surface plasmon nanophotonics enables to investigate light - matter interaction in deep nanoscale and harness electromagnetic and quantum properties of materials, thus opening pathways for tremendous potential applications. However, imaging optical plasmonic waves on a single nanometer scale is yet a substantial challenge mainly due to size and energy considerations. Here, for the first time, we use Kelvin Probe Force Microscopy (KPFM) under optical illumination to image and characterize plasmonic modes. We experimentally demonstrate unprecedented spatial resolution and measurement sensitivity both on the order of a single nanometer. By comparing experimentally obtained images with theoretical calculation results, we show that KPFM maps may provide valuable information on the phase of the optical near field. Additionally, we propose a theoretical model for the relation between surface plasmons and the material workfunction measured by KPFM. Our findings provide the path for using KPFM for high resolution measurements of optical plasmons, prompting the scientific frontier towards quantum plasmonic imaging on submolecular scales. 
the single nanometer scale. A theoretical model is proposed to define the relation between CPD and SPPs and provide an in-depth understanding of the imaging mechanism. We characterize plasmonic nanoantenna and waveguiding structures achieving measurement sensitivity of only $2 \mathrm{~nm}$. Additionally, we observe that the sign of the CPD can be used to obtain information on the electric field's directionality, towards full vector characterization of SPPs. Figure 1 illustrates the process of SPP characterization with scanning KPFM. The origin of nanoplasmonic sensing with KPFM lies in the fact that KPFM measures the contact potential difference (CPD) between the scanning probe tip and the surface ${ }^{26,33}$. The CPD is closely related with the charge distribution on the surface, as well as with the surface plasmon polaritons ${ }^{34-40}$. When nanometallic structures are illuminated with optical radiation, coherent oscillations of free electrons are excited on the surface. These highly confined optically induced charge density waves preserve the frequency of incident photons and strongly affect the work function in the vicinity of the device. Optically excited variations in the workfuction of CdSe nanowires ${ }^{41}$ and $2 \mathrm{D}$ array of subwavelength apertures ${ }^{42}$ was recently characterized by EFM and KPFM.

\section{Results}

According to the Lorentz model, the motion of electrons in metals is governed by a second order partial differential equation

$$
m\left[\frac{\partial^{2} x}{\partial t^{2}}+\Gamma \frac{\partial x}{\partial t}+\Omega^{2} x\right]=-e E
$$

Here $m$ and $e$ is the electron mass and charge, respectively; $\mathrm{x}$ is the electron displacement from the nucleus $\Gamma=1 / \tau$ is the damping rate, $\Omega$ is the spring constant and $\mathrm{E}$ is the electric field. Assuming time harmonic behavior for the electric field and displacement, neglecting $\Omega$ in the free electron region yields the Drude polarizability:

$$
P=-N e x=-\frac{N e^{2}}{m} \frac{1}{\omega^{2}+i \Gamma \omega} E(x, t)
$$

where $\omega=2 \pi f$ is the optical angular frequency of the electric field. The in plane relative permittivity $\left(\varepsilon_{\|} \equiv \varepsilon_{r}+i \varepsilon_{i}\right)$ can be extracted from Equation 2, using $\varepsilon=1+P / E$. The real and imaginary part of $\varepsilon_{\|}$are attributed intraband and interband transitions, respectively. Extended Drude model which considers the restoration force $(\Omega)$ is a preferred analytical description at the bound electron region. This model achieves excellent agreement for Au within $500 \mathrm{~nm}-$ $1000 \mathrm{~nm}$ wavelength range ${ }^{43}$. Further improvement to the extended Drude model can be obtained by adding terms which carefully describe the critical points $(\mathrm{CP})^{44,45}$. Near the plasma frequency, $\omega \approx \omega_{p}=\sqrt{N e^{2} / m \varepsilon_{0}}$, the relative permittivity is very small $\left(\varepsilon\left(\omega_{p}\right) \rightarrow 0\right)$. Hence, $E(x, t)$ becomes a pure depolarization field with quanta of these charge oscillations are referred to as plasmons. Using the relation between polarization and charge density $\rho_{\text {pol }}=-\nabla \cdot P$, the surface electric potential is calculated by integrating the charge density function over the computational surface

$$
V(r)=\iint \frac{\rho_{p o l}(r)}{4 \pi \varepsilon_{0}\left|r-r^{\prime}\right|} d s^{\prime}
$$

The charge density function may also be obtained by solving the Fock equations using an exchange potential operator ${ }^{46,47}$, a more accurate solution but computationally expensive when obtained numerically. The experimental setup used in this work is depicted in Figure 2a. The sample is illuminated from above by unfocused $\mathrm{He}-$ $\mathrm{Ne}$ laser at wavelength of $633 \mathrm{~nm}\left(\omega=2.98 \cdot 10^{15} \mathrm{r} / \mathrm{s}\right)$ at incident angle of $45^{\circ}$. The light beam is linearly polarized in parallel with the dipole orientation, with the sample is appropriately oriented to avoid the cantilever from blocking the light path to the devices. Since $\omega \gg \Gamma \sim 10^{13}$, the in plane relative permittivity can be written as $\varepsilon_{\|}=\varepsilon_{B}-\omega_{p}^{2} / \omega^{2}+i \omega_{p}^{3} / \omega^{2} \tau$, with $\varepsilon_{B}$ is the contribution of bound electrons and $\omega_{p}$ is the plasma frequency. In our measurements topography and KFPM images are recorded sequentially using the lift mode technique to minimize cross talk between the measurements ${ }^{48}$. To this end, we first acquire the surface topography of a single line in tapping mode and then retrace this topography over the same line at a set lift height from the surface to measure the KPFM signal. To map the workfunction of the sample, we apply both AC voltage $\left(\mathrm{V}_{\mathrm{AC}}\right)$ and a $\mathrm{DC}$ voltage $\left(\mathrm{V}_{\mathrm{DC}}\right)$ to the AFM tip. $\mathrm{V}_{\mathrm{AC}}$ generates oscillating electrical forces between the AFM tip and sample surface, and $V_{D C}$ nullifies the oscillating electrical forces that originated from CPD between tip and sample surface. In our study, the tip - sample distance $(\mathrm{H})$ significantly influence the resolution and sensitivity of the measurements. For small values of $\mathrm{H}$, the KPFM image is dominated by short range forces $\left(\sim \mathrm{H}^{-6}\right)$ like Van der Waals and capillary interactions. However, for larger values of $\mathrm{H}$, long range electrostatic and magnetic interactions $\left(\sim \mathrm{H}^{-2}\right)$ govern the results. Therefore, it is critical to optimize the lift height for high quality imaging. To demonstrate unambiguously that we can measure and image plasmonic waves with nanoscopic resolution and sensitivity we chose to investigate hybrid devices of dipole nanoantennas integrated with metal insulator - metal (MIM) plasmonic waveguides as shown in Figure $2 \mathrm{~b}$. These devices are of prime importance for large variety

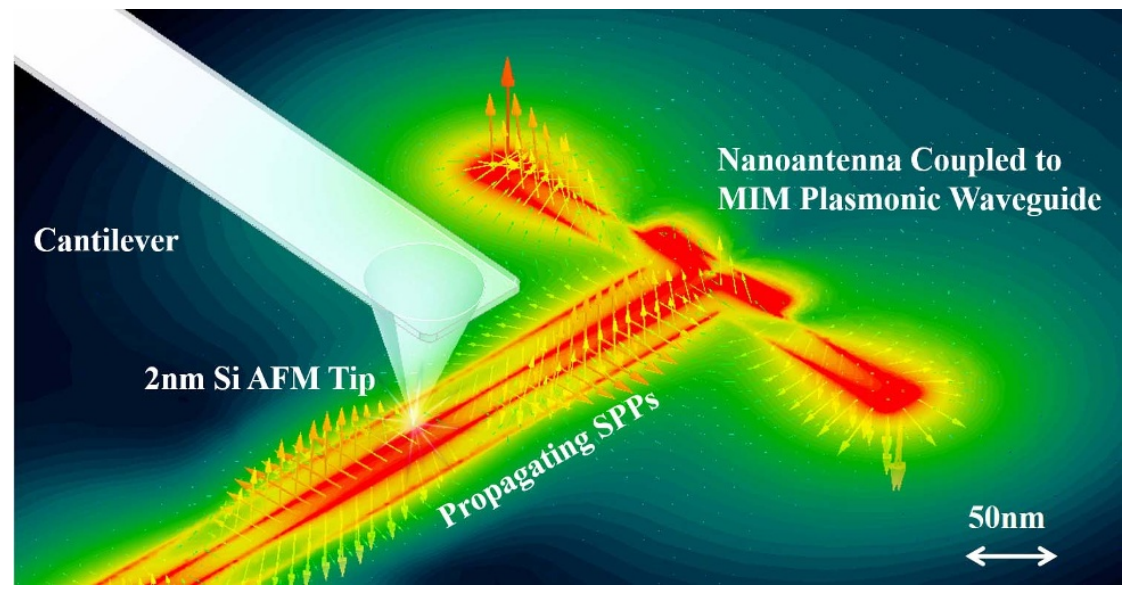

Figure 1 | Illustration of nanoplasmonic characterization with KPFM. Dipole nanoantenna is connected to MIM waveguide with nanometric gap

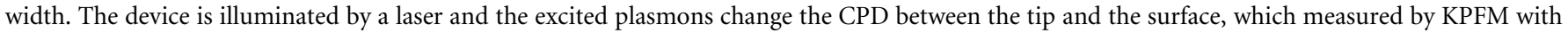
nanometric resolution. 
$\mathbf{a}$

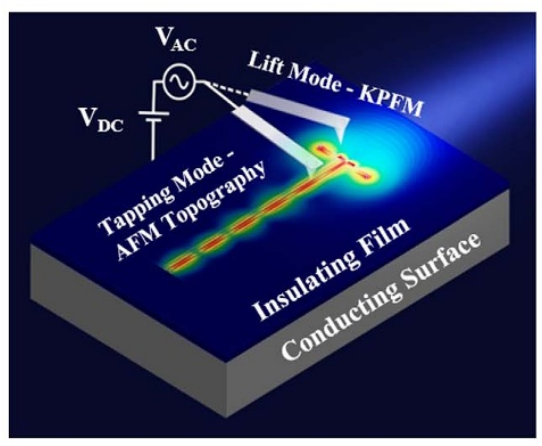

b

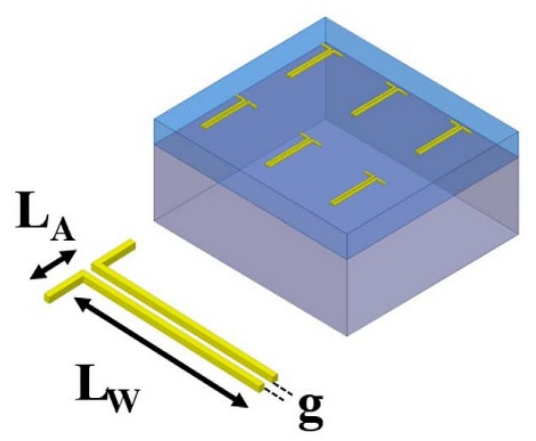

Figure $2 \mid$ Schematics description of our KPFM experimental process and fabricated devices. (a), Schematic of the measurement principle. First the topography is acquired in tapping mode, then the tip elevates to a set lift height, $\mathrm{H}$ to create the CPD map. (b), Description of the analyzed nanoplasmonic devices. Dipole nanoantenna with arm length ' $\mathrm{L}_{\mathrm{A}}$ ' and gap ' $\mathrm{g}$ ', are connected to MIM waveguide of length $\mathrm{L}_{\mathrm{W}}$. The thickness and width of all metal strips are $12 \mathrm{~nm}$.

of applications ${ }^{49,50}$, and have several advantages for this study. First, the combination of nanoantenna and plasmonic waveguide enables to image both localized and propagating SPPs. Localized SPPs are optically excited at the nanoantenna and are coupled to propagating modes along the waveguide. Furthermore, by comparing CPD images with calculated vector fields we show that KPFM measurements may provide information on the vector optical near field. The devices where fabricated by electron beam lithography (EBL), ion beam sputtering (Ag, $10 \mathrm{~nm}$ ) and lift off. By fabricating the structures on the insulating layer of a Si substrate $\left(2 \mu \mathrm{m}\right.$ thick $\left.\mathrm{SiO}_{2}\right)$, we avoid coupling effects between the conducting $\mathrm{Si}$ and the metallic device. After the lift off the PMMA is completely removed, allowing contact between the AFM tip and the metallic devices. Figure 3a presents a scanning electron microscopy (SEM) image of the

a

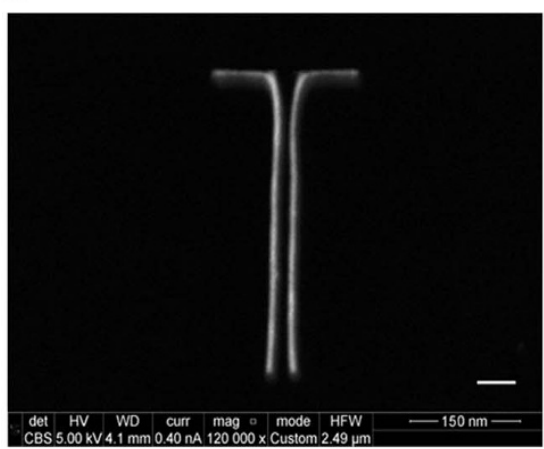

c

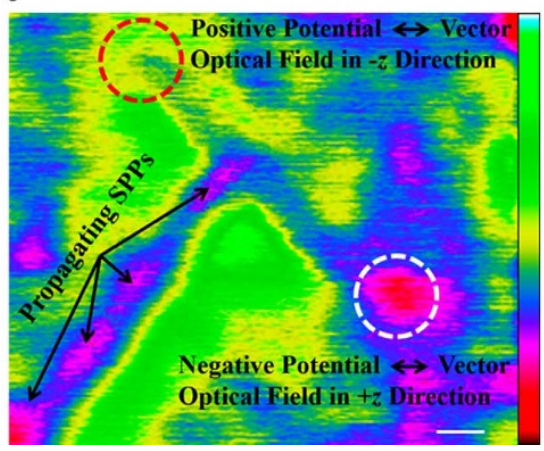

analyzed device, with geometrical dimensions of $\mathrm{L}_{\mathrm{W}}=750 \mathrm{~nm}, \mathrm{~L}_{\mathrm{A}}$ $=100 \mathrm{~nm}$, and $\mathrm{g}=12 \mathrm{~nm}$. The image was recorded at beam current of $0.4 \mathrm{nA}$ and low accelerating voltage of $5 \mathrm{kV}$. Figure $3 \mathrm{~b}$ shows an AFM topography image of the device in 3D, obtained using Si tip with $2 \mathrm{~nm}$ typical diameter. Subsequently, KPFM characterization of the same device was performed in lift mode while the device is illuminated by a He-Ne Laser. The KPFM measurements were performed for tip - sample distances ranging from $10 \mathrm{~nm}-250 \mathrm{~nm}$, with the stage remains spatially fixed and the tip is scanned. We observe that for small values of $\mathrm{H}$, short range interactions strongly affect the KPFM image. For larger tip-sample distances, the results are governed by the long range interactions. The range of distances for which we obtained the best resolution was between $30 \mathrm{~nm}-50 \mathrm{~nm}$. Figure $3 \mathrm{c}$ shows the KPFM image recorded for tip-sample distance of

b

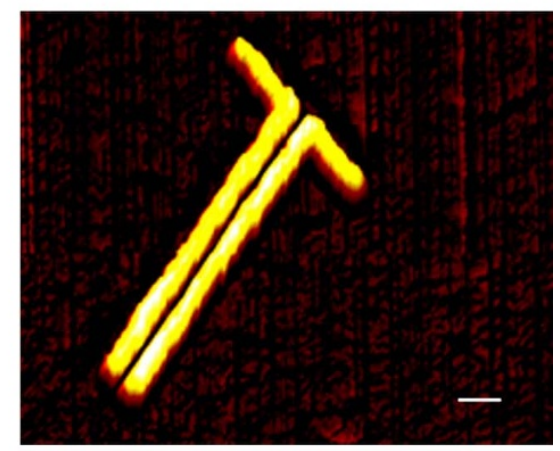

d

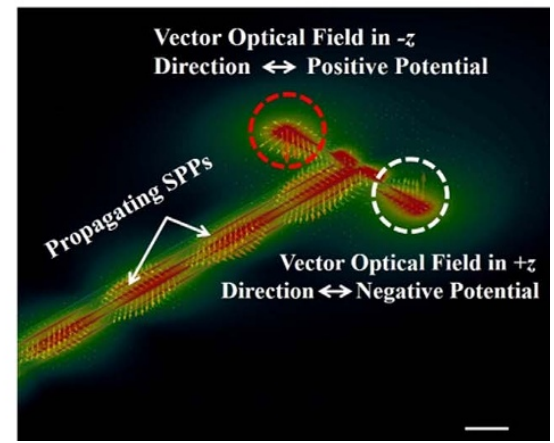

Figure 3 SEM, AFM KPFM and numerical analysis of MIM nanoplasmonic device. (a), High resolution SEM image of the fabricated and analyzed device (b), 3D AFM topography image of the device. The image was recorded with Si super sharp tip with $2 \mathrm{~nm}$ aperture. (c), CPD image of the device under optical illumination with He-Ne laser at frequency of $474 \mathrm{THz}$, recorded with the same tip as (b). KPFM Signal scale bar: $-1.7 \mathrm{~V}-+1.7 \mathrm{~V}$. (d), 3D Numerical calculation (FEM) results of the of the analyzed device under optical illumination of He-Ne laser, similar to the experimental setup used to obtain the results in (c). Scale bar: $50 \mathrm{~nm}$. 

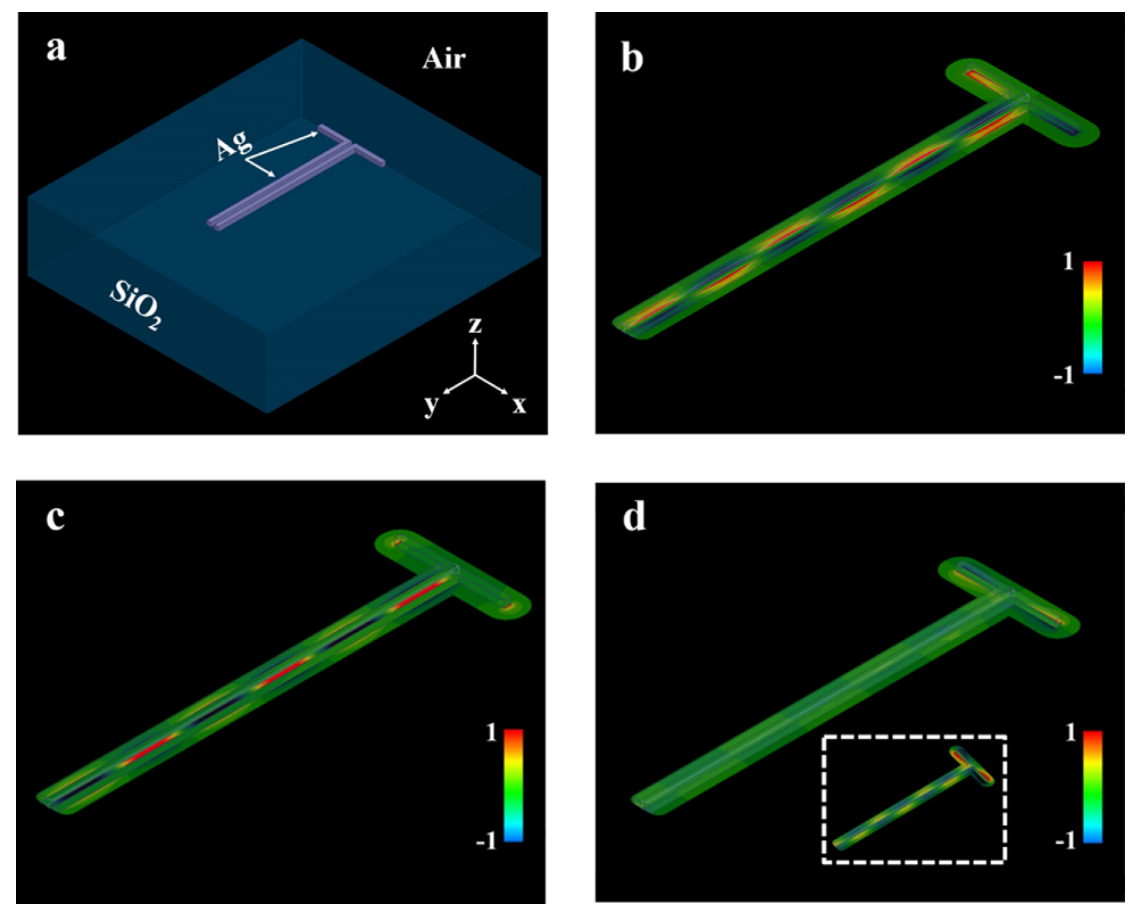

Figure 4 Energy transport and propagation properties of dipole nanoantenna coupled to MIM nanoplamonic waveguide. (a), 3D Schematics of the analyzed devices (b), Numerically calculated near-field image showing $\operatorname{Re}\left(\mathrm{E}_{\mathrm{z}}\right)=\left|\mathrm{E}_{\mathrm{z}}\right| \cos \left(\phi_{\mathrm{z}}\right)$. (c), Numerically calculated near-field image showing Re( $\left.\mathrm{E}_{\mathrm{x}}\right)$ $=\left|\mathrm{E}_{\mathrm{x}}\right| \cos \left(\phi_{\mathrm{x}}\right)$. (d), Numerically calculated near-field image showing $\operatorname{Re}\left(\mathrm{E}_{\mathrm{y}}\right)=\left|\mathrm{E}_{\mathrm{y}}\right| \cos \left(\phi_{\mathrm{y}}\right)$, with the inset shows $\mathrm{E}_{\mathrm{y}}$ for normalized field scale of $\pm 1 / 4$ (instead of \pm 1 ).

$\mathrm{H}=35 \mathrm{~nm}$, which exhibits clear nanometric resolution. We observe two dipolar-like modes on the antenna segments, with strong fields at the antenna ends and at the gap, as expected for a dipole plasmonic nanoantenna in the visible ${ }^{32}$. The localized fields at the nanoantenna gap extend along the MIM waveguide, periodically changing their polarity. The modal behavior of the propagating SPPs along the waveguide is observed in the KPFM image (Fig. 3c). Four periods of modes appear along the waveguide, represented by peaks of the KPFM signal, as the measured ${ }^{32}$ SPP wavelength is $240 \mathrm{~nm}$. The shape of the waveguide, as well as the field decay into its metallic arms are very well captured. This provides direct experimental imaging of a propagating surface plasmon polaritons, obtained for the first time for channel waveguides with $12 \mathrm{~nm} \times 10 \mathrm{~nm}$ gap crossection. Strong localized fields are observed also on the exterior metal - insulator interfaces of the waveguides, as predicted by the theory of SPPs ${ }^{1}$. We observe that the measured KPFM fields on the ends of the nanoantenna are inversely polarized, with the field on the left edge is positive $+1.5 \mathrm{~V}$ and the field on the right edge is negative $-1.5 \mathrm{~V}$, corresponds to local optical near field direction of $+\hat{Z}$ and $-\hat{Z}$, respectively (see Fig. $3 \mathrm{~d}$ ). This behavior is reproduced in numerical calculation results, presented at the optical frequency of $474 \mathrm{THz}$ $(633 \mathrm{~nm})$. The numerical results are obtained using Ansys High Frequency Structure Simulator (HFSS V1 $5^{32,51,52}$ ) based on the finite element method (FEM). Generally, the electric field is described by a three dimensional vector $\mathrm{E}=\left(\mathrm{E}_{\mathrm{x}}, \mathrm{E}_{\mathrm{y}}, \mathrm{E}_{\mathrm{z}}\right)$, where each field component $E_{i}$ is characterized by both magnitude $\left|E_{i}\right|$ and a phase $\phi_{i}{ }^{53}$. Figure $3 \mathrm{~d}$ presents the $3 \mathrm{D}$ electric field vector, $|\mathbf{E}|$, with each arrow represents the direction of the local electric field vector. In spherical coordinates system, the angles $\theta, \varphi$ of each arrow shown in Figure $3 \mathrm{~d}$ are the angles define the direction of the local near field vector. Figure 4 shows the numerically calculated scalar components of the electric fields on the device. Figure $4 \mathrm{~b}$ shows the real part of the vertical near-field component $\operatorname{Re}\left(\mathrm{E}_{\mathrm{z}}\right)=\left|\mathrm{E}_{\mathrm{z}}\right| \cos \left(\phi_{\mathrm{z}}\right)$ where $\left|\mathrm{E}_{\mathrm{z}}\right|$ is the near-field amplitude and $\phi_{\mathrm{z}}$ the phase. We observe high intensity at the nanoantenna ends and gap as well as periodical change in the field polarity, as expected for optical gap nanoantennas ${ }^{17}$. For the horizontal near field component $\mathrm{E}_{\mathrm{x}}$, as shown in Figure 4c. For $\operatorname{Re}\left(\mathrm{E}_{\mathrm{x}}\right)$, we can see that the amplitude image exhibits a completely different pattern, featuring a highly localized, intense fields at the nanoantenna gap and ends with a spatially constant phase ${ }^{17}$. Similarly, for the $\mathrm{E}_{\mathrm{x}}$ near field component, there is no phase difference between the two waveguide arms. Since $E_{x}$ has significantly higher amplitude than $E_{z}$, the phase behavior inside the waveguide is dominated by $E_{x}$. This result explains why no phase difference between the fields inside the waveguide is observed in the KPFM image (Fig. 3c). As expected, the $\mathrm{E}_{\mathrm{y}}$ component of the near field exhibits much lower enhancement, see Figure $4 \mathrm{~d}$. Interestingly, the phase structure of $E_{y}$ may explain the slight shift of the phase center of the KPFM signal (Fig. 3c) with respect to the topography. Figure 5a presents a high resolution AFM image of the fabricated device with gap of $4 \mathrm{~nm}\left(\mathrm{~g}=4 \mathrm{~nm}, \mathrm{~L}_{\mathrm{A}}=20 \mathrm{~nm}\right)$, which sets the lower limit for the gap width achievable via our EBL fabrication process. The KPFM signal of the corresponding topography is shown in Figure 5b. The device exhibits lower field enhancement, which may be attributed to the off resonance dimensions. Additionally, the gap width may approach $\sim 1 \mathrm{~nm}$ at some points due to undesired roughness of the lithography process. This may lead to decreased field enhancement originated from quantum charge transfer ${ }^{54}$. Figure $5 \mathrm{c}$ shows the topography (blue) and KPFM (black) of the device plotted along the dipole axis. We observe that the KPFM signal of the different nanoantenna arms can be clearly resolved, while separated by only $\sim 4 \mathrm{~nm}$ gap. This provides first direct experimental evidence of KPFM capability to image surface plasmon modes with unprecedented resolution on the order of a single nanometer. This imaging resolution of KPFM holds potential for further improvements which can be obtained using sharper tips and process optimization ${ }^{24}$ Now, we demonstrate another substantial vantage of KPFM for plasmonic characterization, by resolving the propagation properties of MIM waveguides with gap (g) difference of $2 \mathrm{~nm}$. Figure 6a presents the configuration of the analyzed devices. Figure $6 \mathrm{~b}$ shows the calculated electric field at frequency of $474 \mathrm{THz}(\lambda=633 \mathrm{~nm}$,) for devices with $\mathrm{L}_{\mathrm{W}}=900 \mathrm{~nm}, \mathrm{~g}=10 \mathrm{~nm}, 20 \mathrm{~nm}, 30 \mathrm{~nm}$ and $60 \mathrm{~nm}$. We observe 

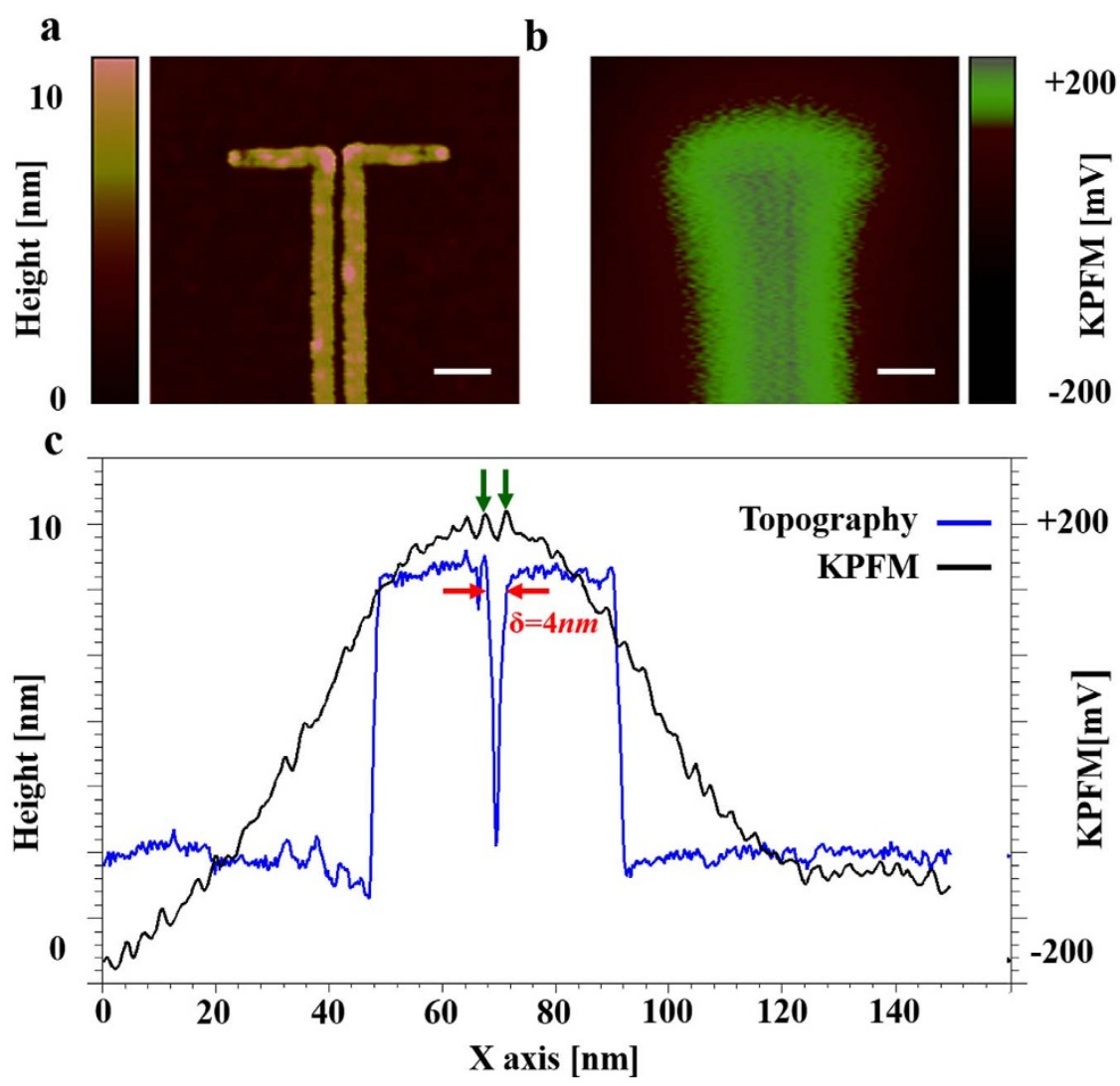

Figure $5 \mid$ Experimental demonstration of KPFM resolution in plasmonic imaging. (a), High resolution AFM image of the device with $g=4 \mathrm{~nm}$, $\mathrm{L}_{\mathrm{A}}=20 \mathrm{~nm}$ (b), Corresponding KPFM map of the same device under optical illumination (c), Experimental topography (blue line) and KPFM (black line) across the nanoantenna axis. Scale bar: $20 \mathrm{~nm}$.

inverse relation between the wavelength of the propagating SPPs, $\lambda_{\mathrm{SPP}}$, and the gap width. As the gap width decreases, the plasmons are confined in both dimensions and vice versa; a confinement which increases the propagation loss. This nonlinear dispersion behavior is evidenced by the difference number of SPP periods propagate within the waveguide. For $\mathrm{g}=10 \mathrm{~nm}$, seven periods of SPP modes are contained inside the waveguide, as for $\mathrm{g}=60 \mathrm{~nm}$ only four periods are observed (see Fig 6b). The calculated electric field along the center of the waveguide (dashed line in Fig. 6a) is shown in Figure 6c, which further demonstrate the change in $\lambda_{\mathrm{SPP}}$ as the gap width increases. Figure $6 \mathrm{~d}$ shows the KPFM signal map for $\mathrm{g}=30 \mathrm{~nm}$ and tip sample distance of $\mathrm{H}=35 \mathrm{~nm}$, selected for maximum resolution. This image has similar characteristics to Figure $3 c$ with two main differences. First, only three periods of SPPs are contained within the waveguide (compared with four periods in Fig. 3c), this is due to the increase in gap width from $12 \mathrm{~nm}$ to $30 \mathrm{~nm}$; a result which is in very good agreement with our calculations (Fig 6b). Second, lower field intensities are observed at the gap and on both nanoantenna ends, mainly caused by impedance mismatch between the dipole and the waveguide and by geometrical shift from resonance. The exact spatial frequency of the propagating SPPs is detected by analyzing the frequency content of the KPFM measurements along the waveguide via spatial Fourier transform (FFT). The black graph in Figure 6e presents the results of spatial FFT performed on the KPFM signal inside the waveguide (marked by line 1 in Fig. $6 \mathrm{~d}$ ), with the blue curve corresponds to spatial FFT performed outside the device (line 2). Both measurements shown in Figure 6e have dominant field component at low frequencies, a result which is in good agreement with the general electrostatic character of KPFM and can be referred to the standing waves in the structure. However, we observe strong spatial frequency component in the field along the device, $\lambda_{\mathrm{SPP}}=$ $248 \mathrm{~nm}$. The amplitude of this component is $15 \mathrm{~dB}$ higher than the corresponding component in the measurement described by the blue curve, providing solid evidence for the existence of propagating SPPs inside the waveguide. We used this method to characterize the propagation properties is a series of fabricated nanoplasmonic devices with gap width varying between $10 \mathrm{~nm}-30 \mathrm{~nm}$ in steps of only $2 \mathrm{~nm}$ (i.e. $\mathrm{g}=10 \mathrm{~nm}, 12 \mathrm{~nm}, \ldots .30 \mathrm{~nm}$ ), $\mathrm{L}_{\mathrm{A}}=100 \mathrm{~nm}$ and $\mathrm{L}_{\mathrm{W}}=3000 \mathrm{~nm}$. For each KPFM measurement, we found $\lambda_{\text {SPP }}$ by performing spatial FFT and measuring the plasmon - wavelength at the peak of the curve (see Fig. 6e).We use long waveguides $\left(\mathrm{L}_{\mathrm{W}} \gg \lambda_{\mathrm{SPP}}\right)$ in order to minimize the standing waves in the device and improve the sensitivity. Figure 7a shows the experimentally obtained dispersion curve (blue graph), with the calculation results presented in the black curve. Real time image of the characterization process via KPFM under laser illumination if presented in Figure $7 \mathrm{~b}$. We observe different experimentally obtained plasmon propagation wavelength $\left(\lambda_{\mathrm{SPP}}\right)$ for waveguides with gap difference of $2 \mathrm{~nm}$, which confirm that KPFM can resolve SPPs with $2 \mathrm{~nm}$ resolution, currently limited by the aperture dimensions of the scanning probe. The maximum deviation between the calculated and experimental dispersion curves is $\delta=2.5 \mathrm{~nm}$, less than $1 \%$ from the mean $\lambda_{\mathrm{SPP}}$.

\section{Discussion}

In Summary, we introduced KPFM as an exceptional method for imaging and characterization of plasmonic modes at optical frequencies. By investigating channel waveguides coupled to nanoantennas, we characterized both surface plasmons and surface plasmon polaritons at the nanoscale. We characterized a variety of nanoplasmonic devices, with experimentally demonstrated spatial resolution and sensitivity both on the order of a single nanometer. The KPFM images include information on the amplitude and phase of the optical near field, and are of good agreement with full wave numerical 


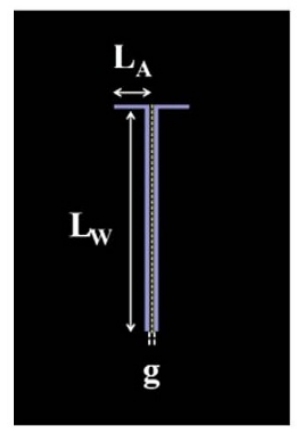

d

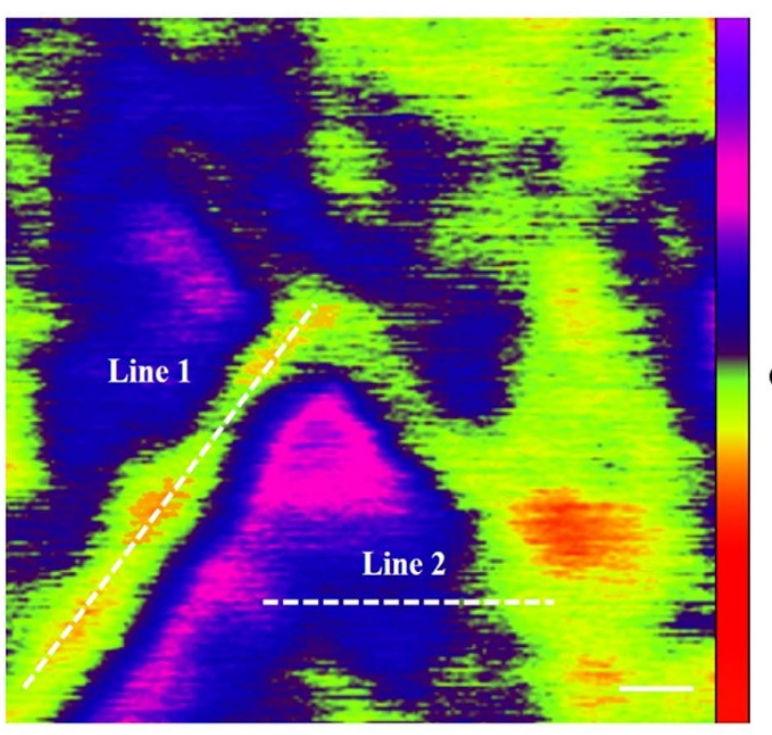

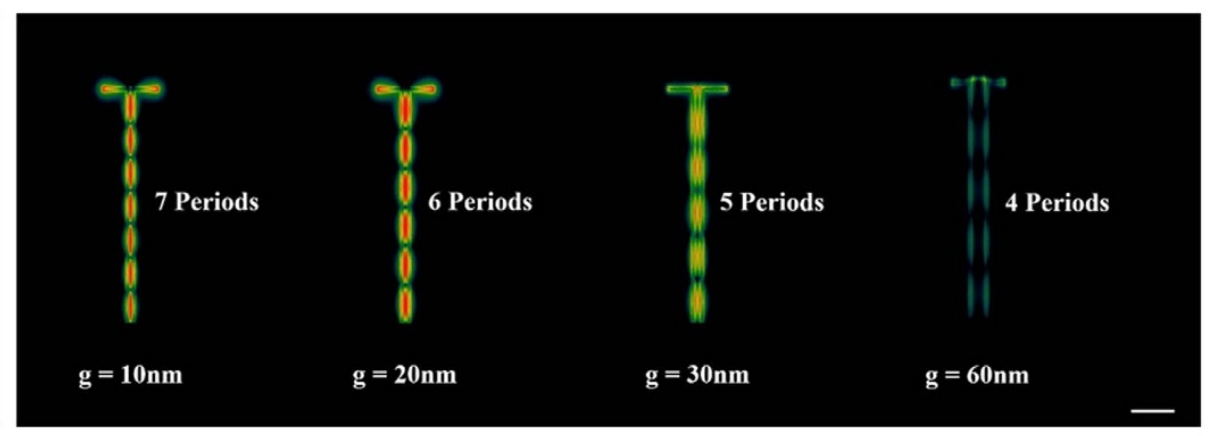

c

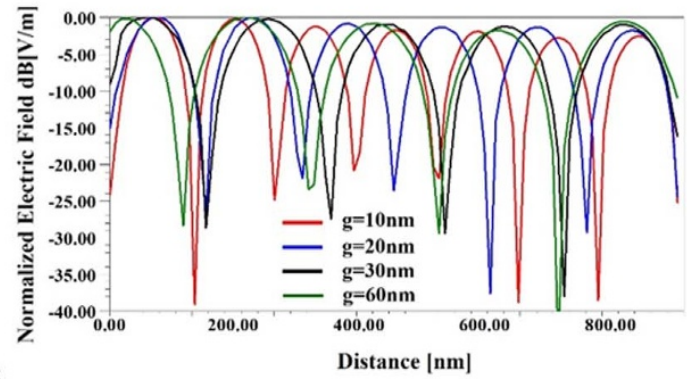

e

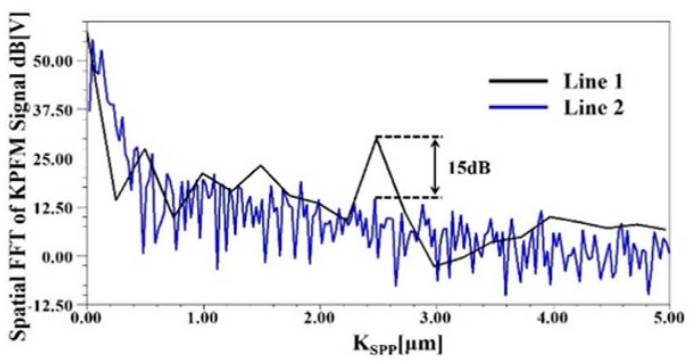

Figure 6 Spatial frequency analysis of the nanoplasmonic devices using KPFM, spatial FFT and numerical calculations. (a), Device configuration. (b), Numerically calculated magnitude of the optical near field, $|\mathrm{E}|$ devices with $\mathrm{g}=10 \mathrm{~nm}, 20 \mathrm{~nm}, 30 \mathrm{~nm}$ and $60 \mathrm{~nm}$, under optical illumination. Decrease in the spatial frequency (higher $\lambda_{\mathrm{SPP}}$ ) is observed when g is increased. (c), Calculated $|\mathrm{E}|$ at $474 \mathrm{THz}$, plotted along the center of the MIM waveguides (dashed line in a) (d), CPD image of the device under optical illumination recorded at H $=35 \mathrm{~nm}$. KPFM Signal scale bar: $\pm 1.2 \mathrm{~V}$ (e), Spatial FFT results of the KPFM signal inside (black graph, calculated along Line 1 in (d)) and outside the waveguide (Line 2 in (d)). Scale bars: $100 \mathrm{~nm}$ in (a) and $50 \mathrm{~nm}$ in (d).

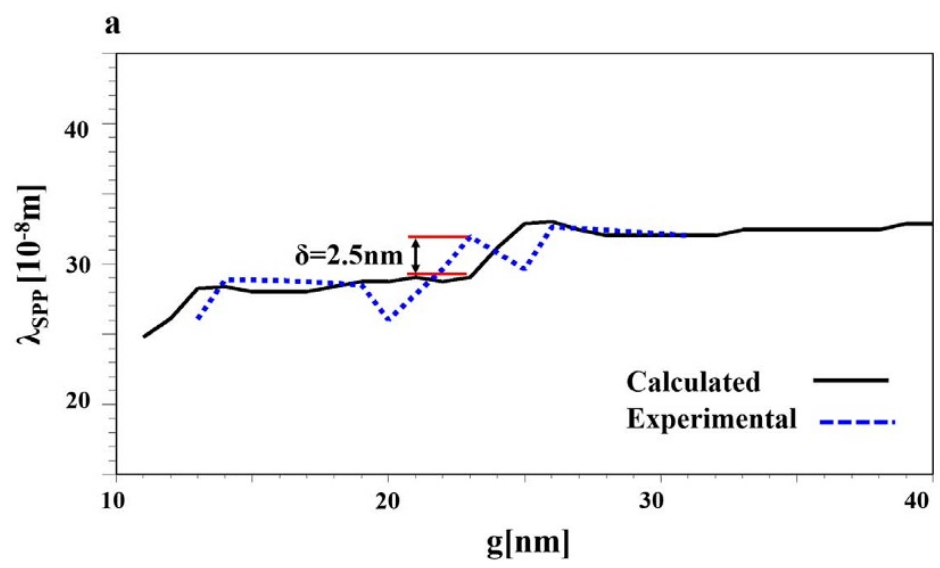

b

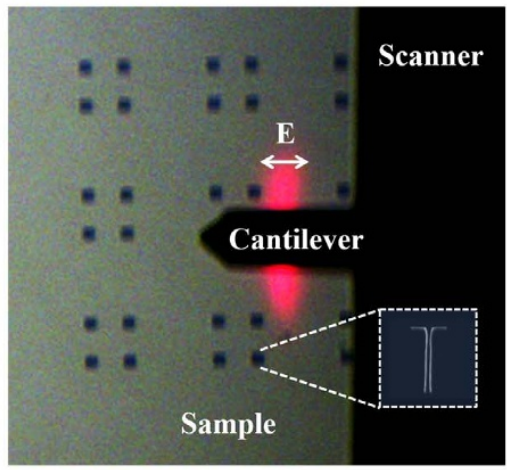

Figure 7 SPP Characterization with $2 \mathrm{~nm}$ resolution. (a), $\lambda_{\mathrm{SPP}}$ as a function of the gap width, g, obtained experimentally (blue dots) and theoretically (continuous black curve). (b), Real time image of the characterization process, captured from the AFM system. Each square includes nanoantennas and MIM waveguide with different gap width. The laser polarization is aligned with the dipole orientation. 
calculations. Our findings open the way for using KPFM under optical illumination for direct imaging of SPPs with nanometric spatial resolution and sensitivity, establishing KPFM as a preferred candidate for experimental characterization of SPP nano devices. It will now be possible for broad community of KPFM users to investigate optically induced charge density waves with dimensions compatible to state of the art future CMOS technology nodes.

\section{Methods}

AFM and KPFM measurements. All measurements were performed at room temperature and free ambient conditions (no vacuum), using Dimension Icon AFM system with NanoScope V controller (Bruker ${ }^{\circledR}$ ). For both AFM and KPFM measurements, we used NanoWorld probes SSS-NCH, SuperSharpSilicon - NonContact/Tapping ${ }^{\mathrm{TM}}$ mode - High resonance frequency; with typical diameter of $2 \mathrm{~nm}$, resonance frequency of $320 \mathrm{kHz}$ and spring constant of $42 \mathrm{~N} / \mathrm{m}$. typically, voltages of $2 \mathrm{~V}$, ac capacitance frequencies of $880 \mathrm{MHz}$, lift heights of $30 \mathrm{~nm}-50 \mathrm{~nm}$ and line rates of $0.1 \mathrm{KHz}$ were employed.

Numerical simulations. The numerical results are obtained by using the software package ANSYS HFSS ${ }^{\mathrm{TM}}$ V15, the industry-standard simulation tool for 3D full-wave electromagnetic field simulation. HFSS solve Maxwell's equations via the finite element method (FEM) using adaptive mesh refinement process for tailored accuracy requirements. The field's solutions are calculated with the metallic (Ag) plasmonic structures being deposited on a homogenous $\mathrm{SiO} 2$ substrate. The nanoantenna is illuminated by optical sources at $474 \mathrm{THz}$ (wavelength of $633 \mathrm{~nm}$ ), which are modeled as focused Gaussian beams with $1 \mu \mathrm{m}$ characteristic diameter. The electric field is polarized in parallel with the dipole direction, as the wave vector $\mathrm{K}$ is perpendicular. A selectively dense meshing is assigned in the metallic and waveguiding regions, with maximum cell size of $1 \mathrm{~nm}$ and 750,000 FEM tetrahedral cells. To provide maximum accuracy, the model is terminated as following: the interface with free space is bounded by perfectly matched layer (PML) absorbing boundary conditions $(\mathrm{ABC})$, while the metallic and $\mathrm{SiO} 2$ termination are done via layered impedance (LI) ABC. The minimum number of adaptive meshing iterations was set to 12 , with convergence condition of $1 \%$ maximum energy variance between adjacent iterations.

Devices Fabrication. SiO2/Si sample was spin-coated with poly (methyl methacrylate) (PMMA 950 A2) electron-beam resist providing thickness of $100 \mathrm{~nm}$. The samples coated with PMMA were subsequently baked for $120 \mathrm{~s}$ on a hotplate at 180C. The desired pattern was exposed in the PMMA layer using a CRESTEC CABLE-9000C high-resolution electron-beam lithography system using different doses to control line and gap width. Then the samples were developed for $90 \mathrm{sec}$ using methyl isobutyl ketone (MIBK), and rinsed with IPA. The samples were subsequently exposed to Ar plasma to etch $10 \mathrm{~nm}$ in order to remove leftovers from the pattern, sputtered using BESTEC 2" DC magnetron to deposit $3 \mathrm{~nm} \mathrm{Cr}$, and $13 \mathrm{~nm} \mathrm{Au}$, then immersed in $180 \mathrm{Khz}$ ultrasonic bath with NMP for $3 \mathrm{~h}$ for resist liftoff.

1. Maier, S. A. Plasmonics: Fundamentals and Application. (Springer, 2007).

2. Schuller, J. A. et al. Plasmonics for extreme light concentration and manipulation. Nat. Mater. 9, 193-204 (2010).

3. Gramotnev, D. K. \& Bozhevolnyi, S. I. Plasmonics beyond the diffraction limit. Nat. Photonics 4, 83-91 (2010).

4. Atwater, H. A. \& Polman, A. Plasmonics for improved photovoltaic devices. Nat. Mater. 9, 205-213 (2010).

5. Berini, P. \& Leon, I. D. Surface plasmon-polariton amplifiers and lasers. Nat. Photonics 6, 16-24 (2012).

6. Graydon, O. Sensing: Plasmonic interferometry. Nat. Photonics 6, 139-139 (2012).

7. Abbe, E. Beiträge zur Theorie des Mikroskops und der mikroskopischen Wahrnehmung. Arch. Für Mikrosk. Anat. 9, 413-418 (1873).

8. Sommerfeld, A. Ueber die Fortpflanzung elektrodynamischer Wellen längs eines Drahtes. Ann. Phys. 303, 233-290 (1899).

9. Heisenberg, W. Über den anschaulichen Inhalt der quantentheoretischen Kinematik und Mechanik. Z. Für Phys. 43, 172-198 (1927).

10. Schnell, M. et al. Nanofocusing of mid-infrared energy with tapered transmission lines. Nat. Photonics 5, 283-287 (2011).

11. Inouye, Y. \& Kawata, S. Near-field scanning optical microscope with a metallic probe tip. Opt. Lett. 19, 159-161 (1994).

12. Betzig, E., Lewis, A., Harootunian, A., Isaacson, M. \& Kratschmer, E. Near Field Scanning Optical Microscopy (NSOM). Biophys. J. 49, 269-279 (1986).

13. Hillenbrand, R., Taubner, T. \& Keilmann, F. Phonon-enhanced light-matter interaction at the nanometre scale. Nature 418, 159-162 (2002).

14. Esteban, R. et al. Direct Near-Field Optical Imaging of Higher Order Plasmonic Resonances. Nano Lett. 8, 3155-3159 (2008).

15. Fei, Z. et al. Gate-tuning of graphene plasmons revealed by infrared nanoimaging. Nature 487, 82-85 (2012).

16. Batson, P. E. Plasmonic Modes Revealed. Science 335, 47-48 (2012).
17. Duan, H., Fernández-Domínguez, A. I., Bosman, M., Maier, S. A. \& Yang, J. K. W. Nanoplasmonics: Classical down to the Nanometer Scale. Nano Lett. 12, 1683-1689 (2012).

18. Scholl, J. A., García-Etxarri, A., Koh, A. L. \& Dionne, J. A. Observation of Quantum Tunneling between Two Plasmonic Nanoparticles. Nano Lett. 13, 564-569 (2013).

19. Iberi, V., Mirsaleh-Kohan, N. \& Camden, J. P. Understanding Plasmonic Properties in Metallic Nanostructures by Correlating Photonic and Electronic Excitations. J. Phys. Chem. Lett. 4, 1070-1078 (2013).

20. Zewail, A. H. Four-Dimensional Electron Microscopy. Science 328, 187-193 (2010).

21. Kim, J. S. et al. Imaging of Transient Structures Using Nanosecond in Situ TEM. Science 321, 1472-1475 (2008).

22. Nonnenmacher, M., O’Boyle, M. P. \& Wickramasinghe, H. K. Kelvin probe force microscopy. Appl. Phys. Lett. 58, 2921-2923 (1991).

23. Mohn, F., Gross, L., Moll, N. \& Meyer, G. Imaging the charge distribution within a single molecule. Nat. Nanotechnol. 7, 227-231 (2012).

24. Grutter, P. Scanning probe microscopy: Seeing the charge within. Nat. Nanotechnol. 7, 210-211 (2012).

25. Jacobs, H. O., Knapp, H. F. \& Stemmer, A. Practical aspects of Kelvin probe force microscopy. Rev. Sci. Instrum. 70, 1756 (1999).

26. Hoppe, H. et al. Kelvin Probe Force Microscopy Study on Conjugated Polymer/ Fullerene Bulk Heterojunction Organic Solar Cells. Nano Lett. 5, 269-274 (2005).

27. Spadafora, E. J., Demadrille, R., Ratier, B. \& Grévin, B. Imaging the Carrier Photogeneration in Nanoscale Phase Segregated Organic Heterojunctions by Kelvin Probe Force Microscopy. Nano Lett. 10, 3337-3342 (2010).

28. Burke, S. A. et al. Determination of the local contact potential difference of PTCDA on NaCl: a comparison of techniques. Nanotechnology 20, 264012 (2009).

29. Sadewasser, S. et al. New Insights on Atomic-Resolution Frequency-Modulation Kelvin-Probe Force-Microscopy Imaging of Semiconductors. Phys. Rev. Lett. 103, 266103 (2009).

30. Nony, L., Foster, A. S., Bocquet, F. \& Loppacher, C. Understanding the atomicscale contrast in Kelvin Probe Force Microscopy. arXiv:0907.4015 (2009). doi:10.1103/PhysRevLett.103.036802.

31. Cohen, M., Zalevsky, Z. \& Shavit, R. Towards Integrated Nanoplasmonic Logic Circuitry. Nanoscale (2013). doi:10.1039/C3NR00830D.

32. Melitz, W., Shen, J., Kummel, A. C. \& Lee, S. Kelvin probe force microscopy and its application. Surf. Sci. Rep. 66, 1-27 (2011).

33. Sommerhalter, C., Matthes, T. W., Glatzel, T., Jäger-Waldau, A. \& Lux-Steiner, M. C. High-sensitivity quantitative Kelvin probe microscopy by noncontact ultrahigh-vacuum atomic force microscopy. Appl. Phys. Lett. 75, 286-288 (1999).

34. Barth, C. \& Henry, C. R. Surface Double Layer on (001) Surfaces of Alkali Halide Crystals: A Scanning Force Microscopy Study. Phys. Rev. Lett. 98, 136804 (2007).

35. Gross, L. et al. Measuring the Charge State of an Adatom with Noncontact Atomic Force Microscopy. Science 324, 1428-1431 (2009).

36. König, T. et al. Measuring the Charge State of Point Defects on $\mathrm{MgO} / \mathrm{Ag}(001)$. J. Am. Chem. Soc. 131, 17544-17545 (2009).

37. Leoni, T. et al. Controlling the Charge State of a Single Redox Molecular Switch. Phys. Rev. Lett. 106, 216103 (2011).

38. Bennett, A. J. Influence of the Electron Charge Distribution on Surface-Plasmon Dispersion. Phys. Rev. B 1, 203-207 (1970).

39. Ekardt, W. Work function of small metal particles: Self-consistent spherical jellium-background model. Phys. Rev. B 29, 1558-1564 (1984).

40. Schäfer, S., Wang, Z., Zierold, R., Kipp, T. \& Mews, A. Laser-Induced Charge Separation in CdSe Nanowires. Nano Lett. 11, 2672-2677 (2011).

41. Hutchison, J. A. et al. Tuning the Work-Function Via Strong Coupling. Adv Mater. 25, 2481-2485 (2013).

42. Vial, A., Grimault, A.-S., Macías, D., Barchiesi, D. \& de la Chapelle, M. L Improved analytical fit of gold dispersion: Application to the modeling of extinction spectra with a finite-difference time-domain method. Phys. Rev. B 71, 085416 (2005).

43. Etchegoin, P. G., Le Ru, E. C. \& Meyer, M. An analytic model for the optical properties of gold. J. Chem. Phys. 125, 164705-164705-3 (2006).

44. Vial, A. \& Laroche, T. Description of dispersion properties of metals by means of the critical points model and application to the study of resonant structures using the FDTD method. J. Phys. Appl. Phys. 40, 7152 (2007).

45. Bardeen, J. Theory of the Work Function. II. The Surface Double Layer. Phys. Rev. 49, 653-663 (1936)

46. Slater, J. C. \& Krutter, H. M. The Thomas-Fermi Method for Metals. Phys. Rev. 47, 559-568 (1935)

47. Jacobs, H. O., Knapp, H. F., Müller, S. \& Stemmer, A. Surface potential mapping: A qualitative material contrast in SPM. Ultramicroscopy 69, 39-49 (1997).

48. Dionne, J. A., Sweatlock, L. A., Atwater, H. A. \& Polman, A. Plasmon slot waveguides: Towards chip-scale propagation with subwavelength-scale localization. Phys. Rev. B 73, 035407 (2006).

49. Fu, Y. et al. All-Optical Logic Gates Based on Nanoscale Plasmonic Slot Waveguides. Nano Lett. 12, 5784-5790 (2012).

50. Vicarelli, L. et al. Graphene field-effect transistors as room-temperature terahertz detectors. Nat. Mater. 11, 865-871 (2012). 
51. Murray, W. A., Suckling, J. R. \& Barnes, W. L. Overlayers on Silver Nanotriangles: Field Confinement and Spectral Position of Localized Surface Plasmon Resonances. Nano Lett. 6, 1772-1777 (2006).

52. Gersen, H., Novotny, L., Kuipers, L. \& van Hulst, N. F. On the concept of imaging nanoscale vector fields. Nat. Photonics 1, 242-242 (2007).

53. Schnell, M., Garcia-Etxarri, A., Alkorta, J., Aizpurua, J. \& Hillenbrand, R. PhaseResolved Mapping of the Near-Field Vector and Polarization State in Nanoscale Antenna Gaps. Nano Lett. 10, 3524-3528 (2010).

54. Savage, K. J. et al. Revealing the quantum regime in tunnelling plasmonics. Nature 491, 574-577 (2012)

\section{Acknowledgments}

M.C acknowledges Olga Girshevitz Yafit Fleger and Yossi Abulafia from Bar-Ilan Institute for Nanotechnology \& Advanced Materials (BINA), for the support in fabricating and characterizing the reported structures.

\section{Author contributions}

M.C. initiated the project, carried out the theoretical design and analysis, designed the devices and the studies, performed the experiments, and wrote the manuscript. R.S. reviewed the manuscript and contributed the computing resources. Z.Z. contributed the experimental facility, reviewed the manuscript and participated in the analysis of the results.

\section{Additional information}

Competing financial interests: The authors declare no competing financial interests.

How to cite this article: Cohen, M., Shavit, R. \& Zalevsky, Z. Observing Optical Plasmons on a Single Nanometer Scale. Sci. Rep. 4, 4096; DOI:10.1038/srep04096 (2014).

(c) (1) (2) This work is licensed under a Creative Commons AttributionBY NA NonCommercial-ShareAlike 3.0 Unported license. To view a copy of this license, visit http://creativecommons.org/licenses/by-nc-sa/3.0 